\title{
Correlation Effects in a One-Dimensional Quarter-Filled Electron System with Repulsive Interactions
}

\author{
Hideo Yoshioka $₫$, Masahisa Tsuchiızu ${ }^{a}$ and Yoshikazu Suzumura ${ }^{a, b}$ \\ ${ }^{a}$ Department of Physics, Nagoya University, Nagoya 464-8602 \\ ${ }^{b}$ CREST, Japan Science and Technology Corporation (JST)
}

(Received December 6, 1999)

\begin{abstract}
A one-dimensional electron system at quarter-filling has been examined by applying the renormalization group method to a bosonized model with on-site $(U)$ and nearest-neighbor $(V)$ repulsive interactions. By evaluating both normal scattering and Umklapp scattering perturbatively, we obtain a phase diagram in which a metallic state with a $2 k_{\mathrm{F}}$ spin density wave $\left(k_{\mathrm{F}}\right.$ is the Fermi wave number) moves into an insulating state with charge disproportionation of a $4 k_{\mathrm{F}}$ charge density wave with an increase in both $U$ and $V$. The effect of the next-nearest-neighbor repulsion is also discussed.
\end{abstract}

KEYWORDS: extended Hubbard model, quarter-filling, commensurability energy, spin density wave, charge density wave

A one-dimensional (1-D) electron system at quarterfilling is a basic model for understanding the electronic properties of quasi 1-D organic conductors 1) The 1-D electron system with only on-site Coulomb repulsive interaction, $U$ (1-D Hubbard model), does not exhibit a metal-insulator (M-I) transition as a function of $U$, and is metallic at any filling, except half-filling. 2 The most dominant state is given by $2 k_{\mathrm{F}}$ spin density wave $\left(2 k_{\mathrm{F}}-\right.$ $\mathrm{SDW}$ ), where $k_{\mathrm{F}}$ is the Fermi wave number. The presence of the long-range repulsive interaction is expected to enrich the phase at quarter-filling. In fact, numerical diagonalization for the model with both $U$ and the nearestneighbor interaction, $V$, 3 , 1 , 6 shows that the insulating phase appears for a large strength of both $U$ and $V$ and that the superconductivity becomes the most dominant fluctuation for a large $V$ and small $U$. On the other hand, several properties have been elucidated within the mean-field theory. 6. With increasing $V$, a transition occurs from a pure $2 k_{\mathrm{F}}$-SDW state to a coexistent state of $2 k_{\mathrm{F}}-\mathrm{SDW}$ and $4 k_{\mathrm{F}}$ charge density wave $\left(4 k_{\mathrm{F}}-\mathrm{CDW}\right)$ d) Such a coexistence is maintained for the charge ordering in (DE-DCNQI $)_{2} \mathrm{Ag}$ observed by the ${ }^{13} \mathrm{C}-\mathrm{NMR}$ measurement 10 The transition has been examined by evaluating the commensurability energy corresponding to the $8 k_{\mathrm{F}^{-}}$ Umklapp scattering The next-nearest-neighbor repulsion results in a coexistence of $2 k_{\mathrm{F}}-\mathrm{SDW}$ and purely electronic $2 k_{\mathrm{F}}-\mathrm{CDW}$ ) and it has been proposed to be the origin of the coexistence gbserved by the X-ray experiment on (TMTSF) ${ }_{2} \mathrm{PF}_{6}$. 11 .

At quarter-filling, the $8 k_{\mathrm{F}}$-Umklapp scattering is crucial to obtain the commensurability energy. Although the above mean-field results may show common features, we need to calculate the Umklapp scattering in the 1-D system by taking into account quantum fluctuation. The existence of commensurability energy of such high order has been pointed out.12, $23,14.15)$ However, to the best of

*E-mail: h44770a@nucc.cc.nagoya-u.ac.jp our knowledge, there are no studies for the phase diagram on the plane of $U$ and $V$, which is calculated by using the analytical expression of the commensurability energy.

In the present paper, a 1-D system with repulsive interactions at quarter-filling is investigated using the bosonization method and the renormalization group (RG) theory. Based on the commensurability energy, 16 a phase diagram is derived on the plane of $U$ and $V$. The relevance of the present results to the observation in (DI-DCNQI $)_{2} \mathrm{Ag}$ salt, 10 and the effect of the nextnearest-neighbor interaction are discussed.

We consider a 1-D extended Hubbard model given by the Hamiltonian, $\mathcal{H}=\mathcal{H}_{0}+\mathcal{H}_{\text {int }}$,

$$
\begin{aligned}
\mathcal{H}_{0} & =-t \sum_{j \sigma}\left(a_{j, \sigma}^{\dagger} a_{j+1, \sigma}+\text { h.c. }\right)-\mu \sum_{j, \sigma} n_{j, \sigma} \\
& =\sum_{K \sigma}\left(\epsilon_{K}-\mu\right) a_{K, \sigma}^{\dagger} a_{K, \sigma}, \\
\mathcal{H}_{\text {int }} & =\frac{U}{2} \sum_{j \sigma} n_{j, \sigma} n_{j,-\sigma}+V \sum_{j \sigma \sigma^{\prime}} n_{j, \sigma} n_{j+1, \sigma^{\prime}} \\
& =\frac{1}{N_{L}} \sum_{\sigma \sigma^{\prime}} \sum_{K_{1} \sim K_{4}}\left\{\frac{U}{2} \delta_{\sigma,-\sigma^{\prime}}+V \mathrm{e}^{-\mathrm{i}\left(K_{2}-K_{3}\right) a}\right\} \\
& \times \delta_{K_{1}+K_{2}-K_{3}-K_{4}, G} a_{K_{1}, \sigma}^{\dagger} a_{K_{2}, \sigma^{\prime}}^{\dagger} a_{K_{3}, \sigma^{\prime}} a_{K_{4}, \sigma},
\end{aligned}
$$

where $t$ and $\mu$ denote the transfer energy and chemical potential, respectively, and $\epsilon_{K}=-2 t \cos K a$ with lattice constant $a$ and $-\pi / a<K \leq \pi / a$. The quantity $a_{j, \sigma}^{\dagger}\left(=1 / \sqrt{N_{L}} \sum_{K} \mathrm{e}^{-\mathrm{i} K a j} a_{K, \sigma}^{\dagger}\right)$ denotes the creation operator of the electron at the $j$-th site with spin $\sigma$, $n_{j, \sigma}=a_{j, \sigma}^{\dagger} a_{j, \sigma}, G=0, \pm 2 \pi / a$, and $N_{L}$ is the number of the lattice. For the later convenience, we divide the one-particle states as $d_{k,-, \sigma}=a_{K, \sigma}$ for $-\pi / a<$ $K \leq-\pi /(2 a), c_{k,-, \sigma}=a_{K, \sigma}$ for $-\pi /(2 a)<K \leq 0$, $c_{k,+, \sigma}=a_{K, \sigma}$ for $0<K \leq \pi /(2 a)$, and $d_{k,+, \sigma}=a_{K, \sigma}$ for $\pi /(2 a)<K \leq \pi / a, k$ being the deviation of the wave 
number from $\pm k_{\mathrm{F}}$ for $c_{k, \pm, \sigma}$, and $\pm 3 k_{\mathrm{F}}$ for $d_{k, \pm, \sigma}$. 18) In terms of $c_{k, p, \sigma}$ and $d_{k, p, \sigma}, \mathcal{H}_{0}$ is written as $\mathcal{H}_{0}=$ $\sum_{p k \sigma}\left\{\left(\epsilon_{p k_{\mathrm{F}}+k}-\mu\right) c_{k, p, \sigma}^{\dagger} c_{k, p, \sigma}+\left(\epsilon_{3 p k_{\mathrm{F}}+k}-\mu\right) d_{k, p, \sigma}^{\dagger} d_{k, p, \sigma}\right\}$ and $\mathcal{H}_{\text {int }}$ is rewritten as $\mathcal{H}_{\text {int }}=\sum_{i=0}^{4} \mathcal{H}_{\text {int, } i}$, where $i$ denotes the number of $d_{k, p, \sigma}$ and/or $d_{k, p, \sigma}^{\dagger}$ in the respective interactions.

When the one-particle states only near the Fermi wave number, $\pm k_{\mathrm{F}}$, are taken into account, the Hamiltonian is reduced to

$$
\begin{aligned}
& \mathcal{H}=\sum_{p k \sigma} p v_{\mathrm{F}} k c_{k, p, \sigma}^{\dagger} c_{k, p, \sigma}+\frac{1}{L} \sum_{p k k^{\prime} q \sigma \sigma^{\prime}} \\
& \left\{\left(g_{1 \perp}^{0} \delta_{\sigma-\sigma^{\prime}}+g_{1 \|}^{0} \delta_{\sigma \sigma^{\prime}}\right) c_{k+q, p, \sigma}^{\dagger} c_{k^{\prime}-q,-p, \sigma^{\prime}}^{\dagger} c_{k^{\prime}, p, \sigma^{\prime}} c_{k,-p, \sigma}\right. \\
& +\left(g_{2 \perp}^{0} \delta_{\sigma-\sigma^{\prime}}+g_{2 \|}^{0} \delta_{\sigma \sigma^{\prime}}\right) c_{k+q, p, \sigma}^{\dagger} c_{k^{\prime}-q,-p, \sigma^{\prime}}^{\dagger} c_{k^{\prime},-p, \sigma^{\prime}} c_{k, p, \sigma} \\
& \left.+\left(g_{4 \perp}^{0} \delta_{\sigma-\sigma^{\prime}}+g_{4 \|}^{0} \delta_{\sigma \sigma^{\prime}}\right) c_{k+q, p, \sigma}^{\dagger} c_{k^{\prime}-q, p, \sigma^{\prime}}^{\dagger} c_{k^{\prime}, p, \sigma^{\prime}} c_{k, p, \sigma}\right\}
\end{aligned}
$$

where $L=N_{L} a$ and the energy dispersion is linearized at $\pm k_{\mathrm{F}}$ as $\epsilon_{ \pm k_{\mathrm{F}}+k}-\mu \simeq \pm v_{F} k$ with $v_{F}=\sqrt{2} t a$. Coupling constants are given by $g_{4 \perp}^{0}=g_{2 \perp}^{0}=U a / 2+V a, g_{4 \|}^{0}=$ $g_{2 \|}^{0}=V a, g_{1 \perp}^{0}=U a / 2$, and $g_{1 \|}^{0}=0$. We note that eq.(3) does not show the M-I transition since there is no Umklapp scattering.

According to a theoretical suggestion by Schulz 13 and calculation of the mean-field theory, 8 Umklapp scattering in the case of quarter-filling appears through the one-particle states with $\pm 3 k_{\mathrm{F}}$, which are connected to those of the Fermi point by $\pm 2 k_{\mathrm{F}}$. We take into account the states near $\pm 3 k_{\mathrm{F}}$ systematically by the following procedure in order to derive the effective Hamiltonian, which consists of the one-particle states only near $\pm k_{\mathrm{F}}$. Representing $c_{k, p, \sigma}$ and $d_{k, p, \sigma}$ in terms of Grassmann algebra, the partition function of eqs.(1) and (2), $Z$, is given by $Z=\int \mathcal{D}\left[d_{k, p, \sigma}^{*} d_{k, p, \sigma}\right] \mathcal{D}\left[c_{k, p, \sigma}^{*} c_{k, p, \sigma}\right] \mathrm{e}^{-S}$, where $S=S_{0}\left[c_{k, p, \sigma}^{*} c_{k, p, \sigma}\right]+S_{0}\left[d_{k, p, \sigma}^{*} d_{k, p, \sigma}\right]+\sum_{i=0}^{4} S_{\mathrm{int}, i}$, and $S_{\text {int, } i}$ denotes the action corresponding to $\mathcal{H}_{\text {int }, i}$. After integrating $d_{k, p, \sigma}$, the partition function, $Z$, is written as $Z=Z_{d}^{0} \int \mathcal{D}\left[c_{k, p, \sigma}^{*} c_{k, p, \sigma}\right] \mathrm{e}^{-S_{\text {eff }}}$, where $Z_{d}^{0}=\int \mathcal{D}\left[d_{k, p, \sigma}^{*} d_{k, p, \sigma}\right] \mathrm{e}^{-S_{0}\left[d_{k, p, \sigma}^{*} d_{k, p, \sigma}\right]}$, and $S_{e f f}=$ $S_{0}\left[c_{k, p, \sigma}^{*} c_{k, p, \sigma}\right]-\ln \left\langle\exp \left(-\sum_{i=0}^{4} S_{\mathrm{int}, i}\right)\right\rangle_{d}\left(\langle\cdots\rangle_{d}\right.$ is the average by $\left.S_{0}\left[d_{k, p, \sigma}^{*} d_{k, p, \sigma}\right]\right)$. By calculating $S_{\text {eff }}$ perturbatively, the effective Hamiltonian is derived where the diagram for the vertex includes only the green functions of $d_{k, p, \sigma}$. From the perturbation up to the second order of $U$ and/or $V$, the correction to the normal processes, $g_{i \|(\perp)}^{0}$, in eq.(3) is calculated as

$$
\begin{aligned}
& \Delta g_{1 \perp}=-4 D_{1}\left(\frac{U a}{2}\right)\left(\frac{U a}{2}-V a\right) \\
& \Delta g_{2 \|}=-2 D_{1}(V a)^{2} \\
& \Delta g_{2 \perp}=-2 D_{1}\left(\frac{U a}{2}\right)^{2}-2 D_{1}\left(\frac{U a}{2}-V a\right)^{2} \\
& \Delta g_{4 \|}=-2 D_{2}(V a)^{2} \\
& \Delta g_{4 \perp}=-2 D_{2}\left(\frac{U a}{2}-V a\right)^{2}
\end{aligned}
$$

where $D_{1}=(8 \pi t a)^{-1} \int_{0}^{\pi / 2} \mathrm{~d} y(\sin y+1 / \sqrt{2})^{-1}=$ $(8 \pi t a)^{-1} \sqrt{2} \ln (\sqrt{2}+1) \simeq 1.25 /(8 \pi t a)$ and $D_{2}=$ $(4 \pi t a)^{-1} \int_{0}^{\pi / 2} \mathrm{~d} y(\sin y+\cos y+\sqrt{2})^{-1}=(8 \pi t a)^{-1} 2 \sqrt{2} /(\sqrt{2}+$ $1) \sim 1.17 /(8 \pi t a)$. The $8 k_{\mathrm{F}}$-Umklapp scattering, which comes from the third order expansion, is obtained as

$$
\begin{aligned}
\mathcal{H}_{1 / 4} & =\frac{(U a)^{2}}{2 t^{2}}(U a-4 V a) \\
& \times \sum_{p} \int \mathrm{d} x\left(\psi_{p,+}^{\dagger} \psi_{-p,+}\right)^{2}\left(\psi_{p,-}^{\dagger} \psi_{-p,-}\right)^{2} \\
& +\frac{(U a)^{3}}{8 t^{2}} \sum_{p \sigma} \int \mathrm{d} x\left(\psi_{p, \sigma}^{\dagger} \psi_{-p, \sigma}\right)^{3} \psi_{p,-\sigma}^{\dagger} \psi_{-p,-\sigma}
\end{aligned}
$$

where the first term corresponds to the conventional commensurability energy 16 ) and $\psi_{p, \sigma}=1 / \sqrt{L} \sum_{k} \mathrm{e}^{\mathrm{i} k x} c_{k, p, \sigma}$. In the third order, there are other contributions leading to the correction to the normal processes, which are disregarded in the following due to eqs.(4)-(8) being large enough for the present choice of parameters.

Here we utilize the bosonization method 19 by introducing phase variables for the charge (spin) fluctuation, $\theta_{\rho}$ and $\phi_{\rho}$, $\left(\theta_{\sigma}\right.$ and $\left.\phi_{\sigma}\right)$, which are defined by $\theta_{\rho(\sigma)}=\sum_{q} \pi \mathrm{i} /(q L) \mathrm{e}^{-\alpha|q| / 2-\mathrm{i} q x} \sum_{k p \sigma}(\sigma) c_{k+q, p, \sigma}^{\dagger} c_{k, p, \sigma}$ and $\phi_{\rho(\sigma)}=\sum_{q} \pi \mathrm{i} /(q L) \mathrm{e}^{-\alpha|q| / 2-\mathrm{i} q x} \sum_{k p \sigma}(\sigma) p c_{k+q, p, \sigma}^{\dagger} c_{k, p, \sigma}$, respectively. The quantity $\alpha^{-1}$ is the ultraviolet cutoff. Those fields satisfy, $\left[\theta_{\nu}(x), \phi_{\nu^{\prime}}\left(x^{\prime}\right)\right]=\mathrm{i} \pi \operatorname{sgn}(x-$ $\left.x^{\prime}\right) \delta_{\nu \nu^{\prime}}(\nu=\rho$ or $\sigma)$. The electron operator is expressed as $\psi_{p, \sigma}=(2 \pi \alpha)^{-1 / 2} \exp \left\{\mathrm{i}(p / 2)\left(\theta_{\rho}+p \phi_{\rho}+\sigma \theta_{\sigma}+\right.\right.$ $\left.\left.\sigma p \phi_{\sigma}\right)\right\} \exp \left(\mathrm{i} \pi \Xi_{p \sigma}\right)$ with $\Xi_{p+}=p / 2 \sum_{p^{\prime}} \hat{N}_{p^{\prime}+}$ and $\Xi_{p-}=$ $p / 2 \sum_{p^{\prime}} \hat{N}_{p^{\prime}-}+\sum_{p^{\prime}} \hat{N}_{p^{\prime}}+$ where $\hat{N}_{p \sigma}=\int \mathrm{d} x \psi_{p \sigma}^{\dagger} \psi_{p \sigma}$. In terms of these phase variables, the Hamiltonian is expressed as $\mathcal{H}=\mathcal{H}_{\rho}+\mathcal{H}_{\sigma}+\mathcal{H}^{\prime}$, where

$$
\begin{aligned}
\mathcal{H}_{\rho} & =\frac{v_{\rho}}{4 \pi} \int \mathrm{d} x\left\{\frac{1}{K_{\rho}}\left(\partial_{x} \theta_{\rho}\right)^{2}+K_{\rho}\left(\partial_{x} \phi_{\rho}\right)^{2}\right\} \\
& +\frac{g_{1 / 4}}{2(\pi \alpha)^{2}} \int \mathrm{d} x \cos 4 \theta_{\rho}, \\
\mathcal{H}_{\sigma} & =\frac{v_{\sigma}}{4 \pi} \int \mathrm{d} x\left\{\frac{1}{K_{\sigma}}\left(\partial_{x} \theta_{\sigma}\right)^{2}+K_{\sigma}\left(\partial_{x} \phi_{\sigma}\right)^{2}\right\} \\
& +\frac{g_{1 \perp}}{(\pi \alpha)^{2}} \int \mathrm{d} x \cos 2 \theta_{\sigma}, \\
\mathcal{H}^{\prime} & =\frac{g_{1 / 4}^{\prime}}{2(\pi \alpha)^{2}} \int \mathrm{d} x \cos 4 \theta_{\rho} \cos 2 \theta_{\sigma} .
\end{aligned}
$$

Here $K_{\nu}=\sqrt{B_{\nu} / A_{\nu}}$ and $v_{\nu}=v_{\mathrm{F}} \sqrt{B_{\nu} A_{\nu}}(\nu=\rho$ or $\sigma)$, where $A_{\rho(\sigma)}=1+\left\{g_{4 \|}+(-) g_{4 \perp}+g_{2 \|}+(-) g_{2 \perp}-\right.$ $\left.g_{1 \|}\right\} /\left(\pi v_{\mathrm{F}}\right)$ and $B_{\rho(\sigma)}=1+\left\{g_{4 \|}+(-) g_{4 \perp}-g_{2 \|}-\right.$ $\left.(+) g_{2 \perp}+g_{1 \|}\right\} /\left(\pi v_{\mathrm{F}}\right)$ with $g_{i \|(\perp)}=g_{i \|(\perp)}^{0}+\Delta g_{i \|(\perp)}$. In eqs.(10)-(12), the coefficients of the nonlinear terms are respectively given by $g_{1 \perp}=g_{1 \perp}^{0}+\Delta g_{1 \perp}=(U a / 2)\{1-$ $\left.4 D_{1}(U a / 2-V a)\right\}, g_{1 / 4}=(U a)^{2}(U a-4 V a) /\left(2 \pi v_{\mathrm{F}}\right)^{2} \times$ $(a / \alpha)^{2}$ and $g_{1 / 4}^{\prime}=(U a)^{3} /\left(2 \pi v_{\mathrm{F}}\right)^{2} / 2 \times(a / \alpha)^{2}$. For $V=0$, the quantity $g_{1 / 2}$ is essentially the same as that reported by Schuld13) and Yonemitsu.15) A contribution from the fourth order of $U$ has also been suggested by Giamarchi 14) The order parameters of the $2 k_{\mathrm{F}}-\mathrm{SDW}$, 
$2 k_{\mathrm{F}}-\mathrm{CDW}$ and $4 k_{\mathrm{F}}-\mathrm{CDW}$ are given by

$$
\begin{aligned}
O_{2 k_{\mathrm{F}}-\mathrm{SDW}} & =\sum_{p \sigma} \sigma \mathrm{e}^{-\mathrm{i} 2 p k_{\mathrm{F}} x} \psi_{p, \sigma}^{\dagger} \psi_{-p, \sigma} \\
& \propto \sin \left(2 k_{\mathrm{F}} x+\theta_{\rho}\right) \sin \theta_{\sigma}, \\
O_{2 k_{\mathrm{F}}-\mathrm{CDW}} & =\sum_{p \sigma} \mathrm{e}^{-\mathrm{i} 2 p k_{\mathrm{F}} x} \psi_{p, \sigma}^{\dagger} \psi_{-p, \sigma} \\
& \propto \cos \left(2 k_{\mathrm{F}} x+\theta_{\rho}\right) \cos \theta_{\sigma}, \\
O_{4 k_{\mathrm{F}}-\mathrm{CDW}} & =\sum_{p} \mathrm{e}^{-\mathrm{i} 4 p k_{\mathrm{F}} x} \psi_{p,+}^{\dagger} \psi_{p,-}^{\dagger} \psi_{-p,-} \psi_{-p,+} \\
& \propto \cos \left(4 k_{\mathrm{F}} x+2 \theta_{\rho}\right) .
\end{aligned}
$$

We investigate the possible states in the limit of low energy. Since the scaling dimension of $\mathcal{H}^{\prime}$ given by $2-8 K_{\rho}-2 K_{\sigma}$ is smaller than that of the other nonlinear terms, the term may be safely neglected for determining the phase diagram. In this case, the Hamiltonian is divided into the charge part and spin part. For the spin part, as long as $g_{1 \perp}>0$, the quantities, $g_{1 \perp}$ and $K_{\sigma}$ tend toward 0 and 1 , respectively, for the low energy limit, due to $S U(2)$ symmetry, and the excitation is gapless. Then the long range correlation functions are given as $\left\langle\sin \theta_{\sigma}(x) \sin \theta_{\sigma}(0)\right\rangle_{\tilde{R}} \tilde{x}^{-1} \ln ^{1 / 2}(x)$ and $\left\langle\cos \theta_{\sigma}(x) \cos \theta_{\sigma}(0)\right\rangle \sim x^{-1} \ln ^{-3 / 2}(x)$ 22) The low energy property for the charge part is determined by the following RG equations,

$$
\begin{aligned}
\frac{d}{d l} K_{\rho}(l) & =-8 G_{1 / 4}^{2}(l) K_{\rho}^{2}(l), \\
\frac{d}{d l} G_{1 / 4}(l) & =\left[2-8 K_{\rho}(l)\right] G_{1 / 4}(l),
\end{aligned}
$$

with the initial conditions given by $K_{\rho}(0)=K_{\rho}$ and $G_{1 / 4}(0)=g_{1 / 4} /\left(2 \pi v_{\rho}\right)$. Here $l=\ln \left(\alpha^{\prime} / \alpha\right)$ with the new length scale $\alpha^{\prime}$ larger than $\alpha$. The solution of the above $\mathrm{RG}$ equations is obtained by

$$
G_{1 / 4}^{2}(l)-1 /\left(2 K_{\rho}(l)\right)-2 \ln K_{\rho}(l)=\text { const },
$$

with a decrease of $K_{\rho}$ with increasing $l$.

The RG flows are shown in Fig.1, where the value of $\alpha \simeq 2 a / \pi$ is used.21) In the regions (I) and (II), the nonlinear term becomes irrelevant, indicating a metallic state. The correlation functions with long distance are given by $\left\langle\sin \theta_{\rho}(x) \sin \theta_{\rho}(0)\right\rangle \sim\left\langle\cos \theta_{\rho}(x) \cos \theta_{\rho}(0)\right\rangle \sim$ $x^{-K_{\rho}(\infty)}$ and $\left\langle\cos 2 \theta_{\rho}(x) \cos 2 \theta_{\rho}(0)\right\rangle \sim x^{-4 K_{\rho}(\infty)}$. Therefore the dominant state exhibits a crossover between $2 k_{\mathrm{F}^{-}}$ SDW and $4 k_{\mathrm{F}}-\mathrm{CDW}$ at $\left.K_{\rho}(\infty)=1 / 322\right)$ where $K_{\rho}(\infty)$ is determined by $G_{1 / 4}^{2}(0)-1 /\left(2 K_{\rho}(0)\right)-2 \ln K_{\rho}(0)=$ $-1 /\left(2 K_{\rho}(\infty)\right)-2 \ln K_{\rho}(\infty)$. The phase boundary determined by $K_{\rho}(\infty)=1 / 3$ is shown by the dashed curve. Both regions, (III) and (IV) are insulating states where $G_{1 / 4} \rightarrow-\infty$ and $\theta_{\rho}$ is locked as $0, \pi / 2 \bmod \pi$ in (III), and $G_{1 / 4} \rightarrow \infty$ and $\theta_{\rho}= \pm \pi / 4 \bmod \pi$ in (IV). The ordered state of $4 k_{\mathrm{F}}-\mathrm{CDW}$ leading to charge ordering is realized in (III) and $\theta_{\rho}=0\left(\theta_{\rho}=\pi / 2\right)$ corresponds to the state in which the charge is rich at the even (odd) sites. The closed circle, open circle and closed square correspond to the initial condition of $V / t=0,2$ and 6 , respectively, with $U / t=5$, showing that the metallic state with $2 k_{\mathrm{F}-\mathrm{SDW}}$ in (I) moves to the insulating state

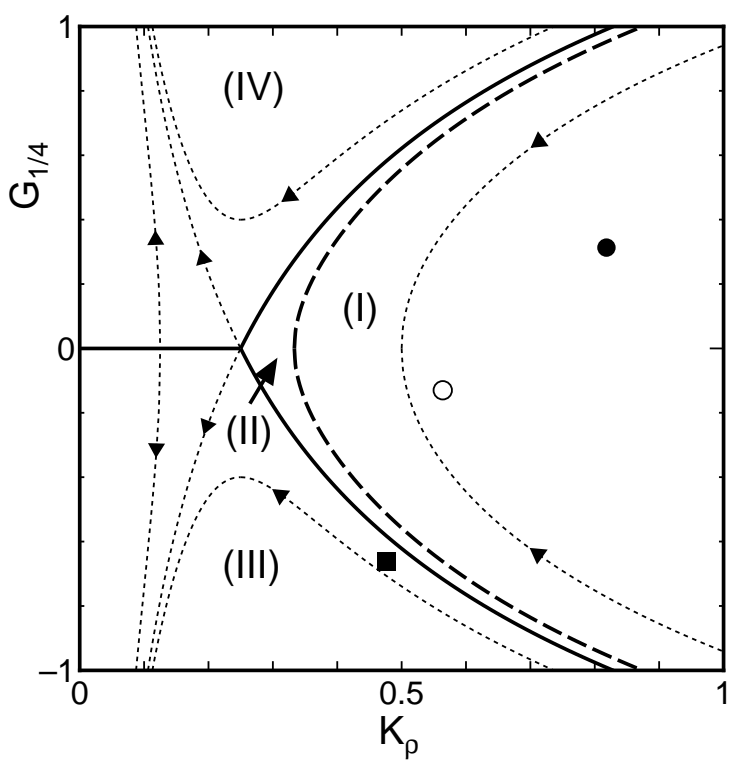

Fig. 1. Scaling flows ( dotted curves ) derived from eqs. (16) and (17) on the plane of $K_{\rho}$ and $G_{1 / 4}$, where the solid and dashed curves denote their boundaries. The parameters in regions (I) and (II) lead to weak coupling, where $K_{\rho}(\infty)=1 / 3$ on the dashed curve. A strong coupling regime is found in both regions, (III) and (IV). The closed circle, open circle and closed square correspond to the initial values of $V / t=0,2$ and 6 , respectively, with the fixed $U / t=5$.

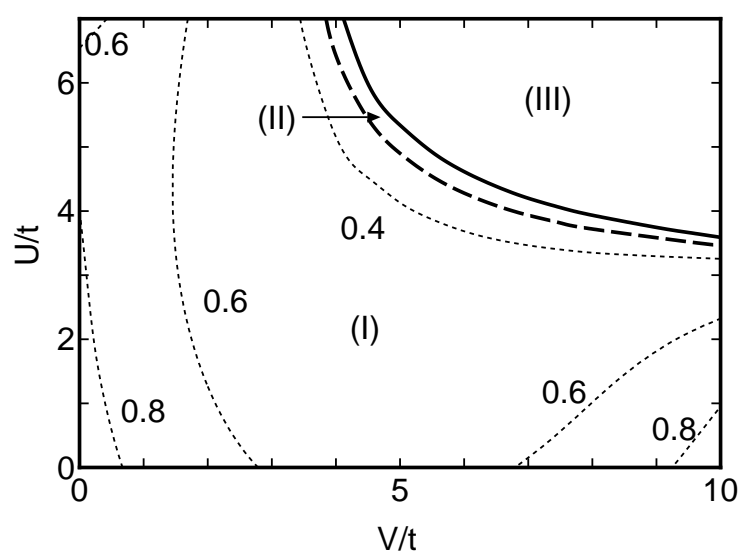

Fig. 2. The phase diagram on the plane of $V / t$ and $U / t$, where regions (I), (II) and (III) separated by boundaries (solid and dashed curves) correspond to those in Fig. 1. The metallic states with $2 k_{\mathrm{F}}-\mathrm{SDW}$ and $4 k_{\mathrm{F}}-\mathrm{CDW}$ are obtained in regions (I) and (II), respectively, while region (III) is the insulating state with $4 k_{\mathrm{F}}$ charge ordering. The dotted curves in the metallic state denote $K_{\rho}(\infty)$.

with the $4 k_{\mathrm{F}}-\mathrm{CDW}$ ordering with increasing $V$.

The phase diagram on the plane of $U / t$ and $V / t$ is shown in Fig.2. The insulating phase appears for a large strength of $U$ and $V$. In addition, for large $V$ and small $U, K_{\rho}(\infty)$ approaches unity indicating that the superconducting fluctuation is enhanced in this region. Figure 2 is qualitatively the same as that derived from the numerical diagonalization. 4 , E 5 In the insulating phase, which corresponds to the region (III) in Fig.1, the $4 k_{\mathrm{F}^{-}}$ CDW, i.e., charge disproportionation, is realized. The state is consistent with the mean-field result 0 ) Thus, the insulating state obtained by the numerical diagonaliza- 
tion is due to the formation of charge disproportionation, which originates in both $U$ and $V$ interactions. Here, we discuss the effects of the nonlinear term, $\cos 4 \theta_{\rho} \cos 2 \theta_{\sigma}$ of eq.(12), which may give minor changes on the phase boundary. In the insulating region, the term seems to give the correction of $g_{1 \perp}$ as $g_{1 \perp} \rightarrow g_{1 \perp}+g_{1 / 4}^{\prime}\left\langle\cos 4 \theta_{\rho}\right\rangle / 2$ with $\left\langle\cos 4 \theta_{\rho}\right\rangle>0$. Such a coupling constant gives rise to the spin gap. However, the procedure breaks the $S U(2)$ symmetry obviously. Therefore, it is expected that the third order correction of $K_{\sigma}$, which has been discarded in the present treatment, would restore the symmetry, and the spin excitation remains gapless in the insulating region.

Within the present analysis, the insulating state exhibits the fluctuation of $2 k_{\mathrm{F}}-\mathrm{SDW}$, while $4 k_{\mathrm{F}}-\mathrm{CDW}$ exists as the true long range order. When three dimensionality is introduced by the interchain coupling, the fluctuation of $2 k_{\mathrm{F}}-\mathrm{SDW}$ becomes the true order. However, the characteristic temperature of $2 k_{\mathrm{F}}-\mathrm{SDW}$ is expected to be smaller than that of $4 k_{\mathrm{F}}-\mathrm{CDW}$. This conclusion may be relevant to the following experimental observation in (DI-DCNQI $)_{2} \mathrm{Ag}$. The magnetic order of $2 k_{\mathrm{F}^{-}}$ SDW is observed at $5.5 \mathrm{~K}$, whereas the resistivity shows insulating behavior at least below room temperature.17 In addition, charge disproportionation is observed below 220 K.10)

Finally, the effects of the next-nearest-neighbor repulsion, $V_{2}$, are briefly discussed. Up to the second order perturbation in the present formulation, the properties of the charge fluctuation do not show a qualitative change. On the other hand, the coefficient of the nonlinear term of the spin fluctuation, $g_{1 \perp}$, is changed as $\left(U a / 2-V_{2} a\right)\left\{1-4 D_{1}\left(U a / 2-V a+V_{2} a\right)\right\}$. Since the nonlinear term with positive (negative) $g_{1 \perp}$ favors $2 k_{\mathrm{F}}-\mathrm{SDW}$ $\left(2 k_{\mathrm{F}}-\mathrm{CDW}\right)$, it is obvious that the repulsive next-nearestneighbor interaction stabilizes $2 k_{\mathrm{F}}$-CDW. In fact, the spin excitation becomes gapped and $2 k_{\mathrm{F}}-\mathrm{CDW}$ is realized when $U / 2<V_{2}$, independent of the strength of $V$ as seen in the mean-field result 23) Thus the quantity $V_{2}$ stabilizes (suppresses) $2 k_{\mathrm{F}}-\mathrm{CDW}\left(2 k_{\mathrm{F}}-\mathrm{SDW}\right)$. However, the coexistence of two kinds of $2 k_{\mathrm{F}}$ density waves, which is predicted by the mean-field theory, $b^{\text {s }}$ cannot be understood from such a calculation, which needs further exploration.

In conclusion, we investigated both normal scattering and Umklapp scattering analytically for one-dimensional quarter-filled electron systems with on-site and nearestneighbor interactions. We have shown that the increase of both $U$ and $V$ leads to the insulating state with charge disproportionation and that the next-nearest-neighbor interaction suppresses (enhances) the fluctuation of $2 k_{\mathrm{F}^{-}}$ $\mathrm{SDW}\left(2 k_{\mathrm{F}}-\mathrm{CDW}\right)$.

\section{Acknowledgment}

The authors would like to thank H. Fukuyama for his useful comments. This work was supported by a Grant-in-Aid for Scientific Research from the Ministry of Education, Science, Sports and Culture (No.11740196) and by a Grant-in-Aid for Scientific Research from the Ministry of Education, Science, Sports and Culture
(No.09640429).

[1] For review, see, D. Jérome and H.J. Schulz: Adv. Phys. 31 (1982) 299, T. Ishiguro, K. Yamaji and G. Saito: Organic Superconductors (Springer-Verlag, Berlin, 1998).

[2] E. H. Lieb and F. Y. Wu: Phys. Rev. Lett. 20 (1968) 1445.

[3] F. Mila and X. Zotos: Europhys. Lett 24 (1993) 133.

[4] K. Penc and F. Mila: Phys. Rev. B 49 (1994) 9670.

[5] K. Sano and Y. Ōno: J. Phys. Soc. Jpn. 63 (1994) 1250.

[6] M. Nakamura, A. Kitazawa and K. Nomura: Phys. Rev. B 60 (1999) 7850.

[7] H. Seo and H. Fukuyama: J. Phys. Soc. Jpn. 66 (1997) 1249.

[8] Y. Suzumura: J. Phys. Soc. Jpn. 66 (1997) 3244.

[9] N. Kobayashi, M. Ogata and K. Yonemitsu: J. Phys. Soc. Jpn. 67 (1998) 1098.

[10] K. Hiraki and K. Kanoda: Phys. Rev. Lett. 80 (1998) 4737.

[11] J. P. Pouget and S. Ravy: J. Phys. I France 6 (1996) 1501, Synth. Met. 85 (1997) 1532.

[12] T. Giamarchi and A. J. Millis: Phys. Rev. B 46 (1992) 9325.

[13] H. J. Schulz: in Strongly Correlated Electronic Materials, eds. K. S. Bedell, Z. Wang, D. E. Meltzer, A. V. Balatsky and E. Abraham (Addison-Wesley Publish Company, 1994) p. 187.

[14] T. Giamarchi: Physica B 230-232 (1997) 975.

[15] K. Yonemitsu: Phys. Rev. B 56 (1997) 7262.

[16] T. Tsuchiizu, H. Yoshioka and Y. Suzumura: Proc. Int. Conf. 22nd Low Temp. Phys., Helsinki 1999, to be published in Physica B.

[17] K. Hiraki and K. Kanoda: Phys. Rev. B 54 (1996) R17276.

[18] K. Penc and F. Mila: Phys. Rev. B 50 (1994) 11429.

[19] V. J. Emery: in Highly Conducting One-Dimensional Solids, eds. J. Devreese, R. Evrard and V. van Doren (Plenum, New York, 1979) p. 247.

[20] T. Giamarchi and H. J. Schulz: Phys. Rev. B 39 (1989) 4620.

[21] This relation can be derived by comparing the response functions near $q=2 k_{\mathrm{F}}$ in the non-interacting case, which are calculated by the conventional fermionic method and by the bosonization method.

[22] H. J. Schulz: Phys. Rev. Lett. 64 (1990) 2831.

[23] Y. Tomio and Y. Suzumura: to be published in J. Phys. Soc. Jpn. 69 (2000) No.3. 